\title{
The termination region of high-mass microquasar jets
}

\author{
V. Bosch-Ramon ${ }^{1}$, M. Perucho ${ }^{2}$, and P. Bordas 3,4 \\ 1 Dublin Institute for Advanced Studies, 31 Fitzwilliam Place, Dublin 2, Ireland \\ e-mail: valenti@cp.dias.ie; vbosch@mpi-hd.mpg.de \\ 2 Dept. d'Astronomia i Astrofísica, Universitat de València, C/ Dr. Moliner 50, 46100 Burjassot (València), Spain \\ e-mail: Manel.Perucho@uv.es \\ 3 Institut für Astronomie und Astrophysik, Universität Tübingen, Sand 1, 72076 Tübingen, Germany \\ 4 INTEGRAL Science Data Centre, Université de Genève, Chemin d'Ecogia 16, 1290 Versoix, Switzerland \\ e-mail: pol . bordas@uni-tuebingen.de
}

Received 20 December 2010 / Accepted 16 January 2011

\section{ABSTRACT}

\begin{abstract}
Context. The environment of high-mass X-ray binaries can be characterized either by the supernova remnant that forms these systems or by the wind from the companion massive star. These regions should be tenuous but very hot and surrounded by a dense and cold shocked ISM shell. The interaction between the jet and such a complex medium, also affected by the system proper motion, can lead to very different jet termination structures.

Aims. The evolution of the jet termination regions during the life of a high-mass microquasar is simulated to improve our present understanding of these structures. Also, the evolving emission characteristics are modeled to inform potential observational campaigns of this class of object.

Methods. We performed 2D numerical simulations of jets propagating in different scenarios, corresponding to different epochs after the formation of the high-mass X-ray binary, using the code Ratpenat. We also made simple estimates of the nonthermal emission that could be produced in the jet termination regions.

Results. We find that, in the way through the hot and tenuous medium of the shocked wind/supernova ejecta, the jet suffers recollimation shocks in which it loses part of its thrust and ends in a strong shock inflating a hot cocoon. The jet head propagates with a speed that is similar to the medium sound speed, until it eventually reaches the denser and colder shocked ISM and the unperturbed ISM later on. In these last stages of evolution, the jet is significantly slowed down and can be disrupted. For relatively old sources, the microquasar peculiar velocity becomes important, leading to complete jet destruction. Extended nonthermal radiation can be generated in the jet termination regions, and hard $\mathrm{X}$-rays and $\mathrm{TeV}$ photons are the wavelengths best suited for observing these structures.
\end{abstract}

Key words. X-rays: binaries - ISM: jets and outflows - radiation mechanisms: non-thermal

\section{Introduction}

High-mass X-ray binaries (HMXB) are the parent population of high-mass microquasars (HMMQ), which show the peculiarity of presenting radio jet activity (e.g. Mirabel \& Rodrígues 1999; Ribó 2005). Jets of HMMQs propagate in a medium characterized by the stellar wind of the massive primary star of the system. On the scales of the binary, the stellar wind can significantly affect the dynamics of the jet, trigger shocks suitable for particle acceleration, and under some conditions, disrupt the jet by a combination of asymmetric recollimation shocks and hydrodynamical instabilities (see Perucho \& Bosch-Ramon 2008; and Perucho et al. 2010).

When launched, powerful enough jets can traverse the companion wind, and once at distances from the injection point that are much longer than the separation distance $\left(z \gg d_{\text {orb }}\right.$, where $z$ is the axial coordinate and distance to the compact object), they do not suffer significant interference by the supersonic wind, which comes from behind the jet. At this stage, the jet head propagates with a speed $\sim \sqrt{n_{\mathrm{j}}\left(d_{\text {orb }}\right) / n_{\mathrm{w}}\left(d_{\text {orb }}\right)} v_{\mathrm{j}} \sim 0.1 v_{\mathrm{j}}$ assuming free expansion, where $v_{\mathrm{j}}$ is the initial jet velocity. In this propagation phase, a strong shock is present at the tip of the jet, and particle acceleration may tap a significant fraction of the jet luminosity there.
When the stellar wind is dynamically negligible, the jet can propagate as far and fast as the inertia of the swept ISM permits and, eventually, the jet head is shocked, thereby inflating a wide cocoon that pushes a shell of shocked ISM (Bordas et al. 2009), as in FRII galaxies (e.g. Fanaroff \& Riley 1974; Kaiser $\&$ Alexander 1997). However, for systems hosting OB stars, the dynamics of the jet may be severely affected before reaching the unperturbed ISM.

To better understand the hydrodynamical jet evolution at different stages of an HMXB, we performed 2D simulations of HMMQ jets for three different scenarios: soon after the SNR explosion (case 1), the phase of wind/ISM interaction (case 2), and the phase in which the wind/ISM interaction is affected by the system proper motion (case 3 ). We also estimated the broadband nonthermal emission that may be produced in cases 1 and 2, and predicted their main observational features.

\section{The physical picture}

For a young HMXB, say of age $t_{\text {src }} \sim 3 \times 10^{4} \mathrm{yr}$, with typical ISM densities $n_{\mathrm{ISM}} \sim 1 \mathrm{~cm}^{-1}$ and moderate peculiar velocities $v_{\mathrm{p}} \lesssim 10^{7} \mathrm{~cm} \mathrm{~s}^{-1}$, the system may still be embedded inside an adiabatically expanding $S N R$ (case 1). In such a situation, if a jet is launched, after $\sim 10 \mathrm{yr}$ it will encounter a region of shocked stellar wind confined by the shocked SNR ejecta, which in turn 
drive a strong shock in the ISM. Once in the high-pressure region of the shocked stellar wind, the jet head will be slowed down to medium subsonic velocities $\left(\sim 1000 \mathrm{~km} \mathrm{~s}^{-1}\right.$ for typical jet and wind properties, see below). At farther distances, the jet head will encounter the shocked $S N R$ ejecta, eventually reaching the shocked ISM. Finally, the jet will enter the normal ISM having lost significant amounts of thrust, producing a shock in the ISM and possibly suffering disruption. Intermittent jet activity with a low duty cycle will not clean the way for later ejections, since the sound speed in the shocked wind and the SNR ejecta is high. High enough duty cycles $(\gtrsim 10 \%)$ for the jet to keep the way clean can be understood in terms of an averaged persistent activity. The case of an HMMQ persistent jet interacting with an SNR has a clear example in SS 433 (see Velázquez \& Raga 2000; Zavala et al. 2008).

Older HMXBs have already left the $S N R$, and the stellar winds can directly interact with the ISM (case 2). In this case, the stellar wind inflates a bubble filled with shocked wind material, and this hot bubble drives a shock in the ISM that becomes radiative even for moderate ages of the source $\left(t_{\mathrm{src}} \sim\right.$ $10^{5} \mathrm{yr}$; see Castor et al. 1975). Therefore, a jet launched in this HMXB phase will interact first with the shocked wind bubble, later reaching a thin, dense, and cold shell of shocked ISM. Qualitatively, the situation is similar to the previous case, but now the ISM shell is much denser because of radiative cooling. Eventually, the jet can reach the unperturbed ISM, shocking it. As in case 1 , the jet may eventually be disrupted. Cygnus X-1 could correspond to the situation represented by case 2 (Martí et al. 1996; Gallo et al. 2005; Russell et al. 2007). Since there are no clear signatures of a reverse shock (Bordas et al. 2009) in this source and only the shocked ISM thermal radiation is detected, it may be that the Cygnus X-1 jet head is fully disrupted. A shock driven in the ISM can still develop in such a conditions since the disrupted jet region has much higher pressure than the environment, as simulations of jets of FRI radio galaxies show (Perucho \& Martí 2007; see also Bordas et al. 2011).

If the HMXB age is old enough, say $t_{\mathrm{src}} \sim 10^{6} \mathrm{yr}$, the winddriven ISM shock can be slower than $v_{\mathrm{p}}$. Then, a bow-shaped shock forms, driven by the system proper motion and pointing in its direction (case 3). In this scenario, the shocked ISM material is slowly evacuated in the opposite direction to the system motion, and the shocked wind material is also advected in the same direction but at much faster velocities, similar to the shocked wind sound speed $\left(\sim 10^{3} \mathrm{~km} \mathrm{~s}^{-1}\right)$. If the jet is launched in this stage, for jet directions that are misaligned enough with the binary motion the shocked wind material will impact the jet roughly from the side. At farther distances, the jet will encounter the shocked ISM, roughly as dense and cold as in case 2 . The dense shocked ISM layer plus lateral impact of the shocked wind will stop the advance of the jet and lead to total jet destruction before reaching the unshocked ISM.

Sketches of the scenarios corresponding to cases 1, 2, and 3 are presented in the top, middle, and bottom panels of Fig. 1.

\section{Studying the jet termination}

\subsection{Medium characteristics}

We have adopted an analytical approach for characterizing the medium in which the jet propagates. In the three cases considered here, the ISM density has been fixed to $n_{\mathrm{ISM}}=1 \mathrm{~cm}^{-3}$, with a temperature $T_{\text {ISM }}=10^{4} \mathrm{~K}$, as expected in regions close to massive stars. The stellar wind velocity and the stellar massloss rate have been taken to be $v_{\mathrm{w}}=2 \times 10^{8} \mathrm{~cm} \mathrm{~s}^{-1}$ and

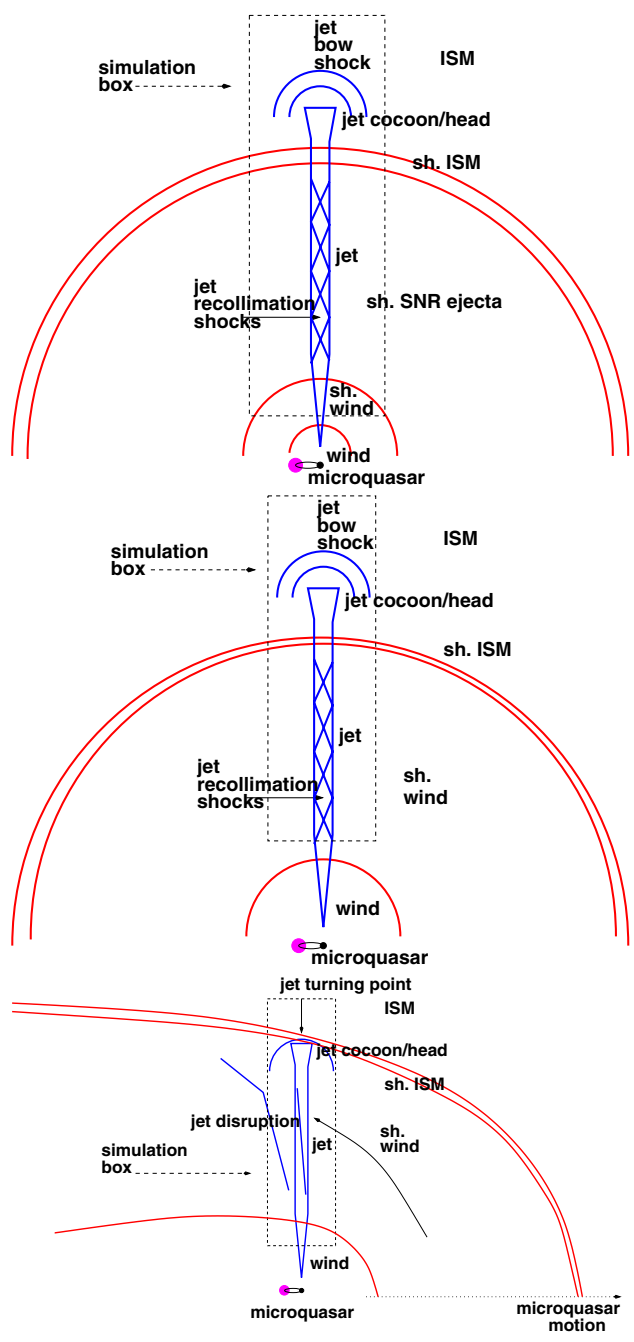

Fig. 1. Sketches of a jet propagating through different media depending on the age of the system: the shocked stellar wind, the shocked SNR ejecta, and the shocked and unshocked ISM (top); the shocked stellar wind, and the shocked and unshocked ISM (middle); through the unshocked and shocked stellar wind, and interacting with the shocked ISM under a significant peculiar velocity (bottom).

$\dot{M}_{\mathrm{w}}=10^{-6} M_{\odot} / \mathrm{yr}$, respectively (typical of an O star; see, e.g., Puls et al. 2009), which corresponds to a kinetic luminosity of the wind $L_{\mathrm{w}}=\dot{M}_{\mathrm{w}} v_{\mathrm{w}}^{2} / 2 \approx 1.3 \times 10^{36} \mathrm{erg} \mathrm{s}^{-1}$.

In case 1 , the system is assumed to be at the center of an $S N R$. The ejected mass of the $S N R$ has been taken to be $2 M_{\odot}$, and the explosion energy $5 \times 10^{49} \mathrm{erg}$. These moderate values have been adopted accounting for the fact that the energetics of supernovae leading to the formation of some HMXBs can be quite low (a relatively extreme case may be Cygnus X-1, see Mirabel \& Rodrigues 2003). We have calculated the properties of the shocked ejecta of the $S N R$ and the shocked ISM by adopting the solution of a spherical explosion in its adiabatic phase (e.g. Blandford \& McKee 1976) for $t_{\text {src }} \sim 3 \times 10^{4} \mathrm{yr}$. The radius of the whole region affected by the $S N R$ is $z_{\mathrm{bs}} \approx\left(E_{\mathrm{SNR}} / m_{\mathrm{H}} n_{\mathrm{ISM}}\right)^{1 / 5} t_{\mathrm{src}}^{2 / 5} \approx 3 \times 10^{19} \mathrm{~cm}$ for the adopted parameter values, where $m_{\mathrm{H}}$ is the hydrogen mass. The density of the shocked ISM is the one for a strong adiabatic shock, $n_{\mathrm{ISMs}}=4 n_{\mathrm{ISM}}$, and the temperature, $T_{\mathrm{ISMs}} \approx 4 \times 10^{5} \mathrm{~K}$. From $z_{\mathrm{bs}}$ and $E_{\mathrm{SNR}}$, we derive a characteristic pressure within that radius, $P_{\mathrm{SNR}} \sim 2 E_{\mathrm{SNR}} / 3 V_{\mathrm{bs}} \approx 3 \times 10^{-10} \mathrm{erg} \mathrm{cm}^{-3}\left(T_{\mathrm{SNR}} \approx\right.$ $\left.10^{8} \mathrm{~K} ; v_{\mathrm{bs}}=4 \pi z_{\mathrm{bs}}^{3} / 3\right)$, as well as the locations of the contact 
Table 1. The ambient medium in cases 1,2 , and 3 .

\begin{tabular}{lccc}
\hline \hline Case 1 & $\begin{array}{c}\text { Jet height } \\
z(\mathrm{~cm})\end{array}$ & $\begin{array}{c}\text { Medium dens. } \\
n\left(m_{\mathrm{p}} \mathrm{cm}^{-3}\right)\end{array}$ & $\begin{array}{c}\text { Medium temp. } \\
T(\mathrm{~K})\end{array}$ \\
\hline Shocked wind & $3 \times 10^{18}$ & 0.02 & $10^{8}$ \\
SNR & $8 \times 10^{18}$ & 0.02 & $10^{8}$ \\
Shocked ISM & $3 \times 10^{19}$ & 4.0 & $4 \times 10^{5}$ \\
ISM & $3.3 \times 10^{19}$ & 1.0 & $10^{4}$ \\
\hline Case 2 & & & \\
Shocked wind & $3 \times 10^{18}$ & $4 \times 10^{-3}$ & $6 \times 10^{7}$ \\
Shocked ISM & $2 \times 10^{19}$ & 100 & $2 \times 10^{3}$ \\
ISM & $2.01 \times 10^{19}$ & 1.0 & $10^{4}$ \\
\hline Case 3 & & & \\
Stellar wind & $1.25 \times 10^{18}$ & $2.4 \times 10^{-4}$ & $10^{3}$ \\
Shocked wind & $8 \times 10^{18}$ & $10^{-3}$ & $10^{8}$ \\
Shocked ISM & $1 \times 10^{19}$ & 100 & $10^{3}$ \\
ISM & $1.01 \times 10^{19}$ & 1.0 & $10^{4}$ \\
\hline
\end{tabular}

discontinuity between the shocked ISM and the shocked ejecta, $z_{\mathrm{c}} \approx 0.9 z_{\mathrm{bs}}$. The location of the contact discontinuity between the shocked stellar wind and the $S N R$ ejecta is computed by taking the same pressure on both sides, obtaining $z_{\mathrm{ws}} \approx 8 \times 10^{18} \mathrm{~cm}$. The shocked wind density is $n_{\mathrm{ws}} \approx 0.02 \mathrm{~m}^{-3}$, and the temperature $T_{\mathrm{ws}} \approx 10^{8} \mathrm{~K}$. We simulate the jet once it is already inside the shocked wind region, since the dynamical impact of the medium on the jet is negligible at closer distances.

In case 2, we have adopted the solution given by Castor et al. (1975) that describes the interaction of a stellar wind with the ISM at the stage when the shock in the latter is already radiative. We adopted an age of $10^{5} \mathrm{yr}$ in this case, and the radius of the whole interaction region is $z_{\mathrm{bs}}=0.76\left(L_{\mathrm{w}} / m_{\mathrm{H}} n_{\mathrm{ISM}}\right)^{1 / 5} t^{3 / 5} \approx$ $2 \times 10^{19} \mathrm{~cm}$. The pressure in the shocked wind region is $P_{\mathrm{ws}} \approx$ $n_{\mathrm{ISM}} m_{\mathrm{H}} \dot{z}_{\mathrm{bs}}^{2} \approx 3 \times 10^{-11} \mathrm{erg} \mathrm{cm}^{-3}\left(T_{\mathrm{ws}} \approx 6 \times 10^{7} \mathrm{~K}\right)$, and the density $n_{\mathrm{ws}}=4 \times 10^{-3} \mathrm{~cm}^{-3}$. The shocked ISM region is a very thin, cool, and dense layer. For simplicity, the width of this region has been taken equal to one simulation cell: $10^{17} \mathrm{~cm}$, with $n_{\mathrm{ISMs}} \approx 10^{2} \mathrm{~cm}^{-3}$ and $T_{\mathrm{ISMs}}=2 \times 10^{3} \mathrm{~K}$. As in case 1 , the jet injection point is already in the wind shocked region.

In case 3 , taking $v_{\mathrm{p}}=10^{7} \mathrm{~cm} \mathrm{~s}^{-1}$ and $t_{\mathrm{src}}=10^{6} \mathrm{yr}$, one gets $\dot{z}_{\mathrm{bs}} \sim 10^{6} \mathrm{~cm} \mathrm{~s}^{-1} \ll v_{\mathrm{p}}$, which means that the winddriven shock in the ISM becomes strongly asymmetric, with the shocked wind material advected backwards with respect to this shock. The wind driven shock in the ISM is now closer to the HMMQ than in case 2 , at $z_{\mathrm{ws}}=10^{19} \mathrm{~cm}$. The shock in the stellar wind is at $z_{\mathrm{w}} \approx 8 \times 10^{18} \mathrm{~cm}$, in which the (pre-shock) stellar wind density and temperature are $n_{\mathrm{w}}=2.4 \times 10^{-4} \mathrm{~cm}^{-3}$ and $T_{\mathrm{w}}=10^{3} \mathrm{~K}$. The density, pressure, and temperature in the shocked wind are $n_{\mathrm{ws}}=10^{-3} \mathrm{~cm}^{-3}, P_{\mathrm{ws}}=1.4 \times 10^{-11} \mathrm{erg} \mathrm{cm}^{-3}$, and $T_{\mathrm{ws}}=10^{8} \mathrm{~K}$, respectively, and a lateral shocked wind velocity of $v_{\mathrm{ws} \perp}=10^{8} \mathrm{~cm} \mathrm{~s}^{-1}$ has been assumed. On the top of the shocked wind region, a thin shocked ISM shell of $10^{17} \mathrm{~cm}$ width, $n_{\mathrm{ISMs}}=100 \mathrm{~cm}^{-3}$, and $T_{\mathrm{ISMs}}=10^{3} \mathrm{~K}$ is located. In this simulation, the jet is launched in the unshocked wind region, because it is more extended than in previous cases, and the unshocked wind is taken as coming from behind the jet. The values of the main ambient medium parameters, for the three cases considered here, are summarized in Table 1.

\subsection{Simulations}

We performed 2D numerical simulations of jets evolving in the three different scenarios described above. In cases 1 and 2 the jets are simulated using an axisymmetric grid in cylindrical coordinates, whereas the transversal motion of the shocked wind in case 3 requires a slab jet in planar coordinates. We expect from our previous experience (see Perucho \& Bosch-Ramon 2008; Perucho et al. 2010) that the results of the slab simulation will be qualitatively correct. The jets are injected with power $L_{\mathrm{j}}=3 \times 10^{36} \mathrm{erg} \mathrm{s}^{-1}$ at $z_{0}=3 \times 10^{18} \mathrm{~cm}$ for cases 1 and 2 , and $z_{0}=1.25 \times 10^{18} \mathrm{~cm}$ for case 3 . In case 3 , the power corresponds to a jet square section (i.e. the power crossing a surface equal to the jet width square). In cases 1 and 2 , the jet is assumed to have a radius at the injection point $R_{\mathrm{j} 0}=0.1 z_{0}$. In case 3 , the jet half-side length has been taken as $1.5 \times 10^{17} \mathrm{~cm}$. We have fixed the jet velocity at $v_{\mathrm{j}}=10^{10} \mathrm{~cm} \mathrm{~s}^{-1}$, the Mach number, $M_{\mathrm{j}} \approx 18$, and the jet temperature, $T_{\mathrm{j}}=1.2 \times 10^{9} \mathrm{~K}$ at injection in all three cases. The jets are composed by protons and electrons and have densities of $\rho_{\mathrm{j}}=1.8 \times 10^{-29} \mathrm{~g} \mathrm{~cm}^{-3}$ for cases 1 and 2 , and $\rho_{\mathrm{j}}=8.3 \times 10^{-29} \mathrm{~g} \mathrm{~cm}^{-3}$ for case 3 . The adiabatic exponent for the jets, with the given temperature and composition is $\Gamma \approx 1.58$. The ambient medium in the different simulations follows the description given in Sect. 3.1. We note that the shock driven by the jet head in the medium (wind, $S N R$, and ISM) is called here bow shock (which is more a sound wave rather than a real shock in the hot shocked wind and SNR ejecta), and the jet shocked region is called cocoon, following Bordas et al. (2009).

The simulations were carried out with the finite-volume code Ratpenat, which solves the equations of relativistic hydrodynamics in conservation form using high-resolution-shockcapturing methods. Ratpenat was parallelized with a hybrid scheme with both parallel processes (MPI) and parallel threads (OpenMP) inside each process (see Perucho et al. 2010). The simulations were performed using the computational facility Tirant, at the "Servei d'Informàtica" of the "Universitat de València", with 12,8 , and 4 processors for cases 1,2 , and 3, respectively. In the axisymmetric simulations, the numerical grid box expands transversally to $100 R_{\mathrm{j} 0}$, with an extended grid of $100 R_{\mathrm{j} 0}$ added to the uniform grid. In the axial direction, the grid extends to $150 R_{\mathrm{j} 0}$ in case 1 , and to $100 R_{\mathrm{j} 0}$ in case 2 . The numerical resolution of these simulation is eight cells per initial jet radius. The computational grid, including the extended region is $1200 \times 1000$ cells. In the case of the simulation of the slab jet, the grid expands $100 R_{\mathrm{j} 0}$ to each side of the axis, plus $100 R_{\mathrm{j} 0}$ extra on each side and $100 R_{\mathrm{j} 0}$ in the axial direction. The resolution used here is four cells per jet initial half-side length. The dimensions of the grid are thus $1200 \times 400$ cells. The boundary conditions adopted in the Cartesian and cylindrical cases are reflection at the jet bottom side, injection right at the jet base, and outflow in the rest. Reflection in the jet axis has been adopted in the cylindrical simulations.

\subsection{Results}

The simulation of case 1 has been run during $8900 \mathrm{yr}$ of evolution. During the first $4500 \mathrm{yr}$, the jet propagates up to $z \approx$ $3 \times 10^{19} \mathrm{~cm}$, with an advance velocity $v_{\mathrm{h}} \approx 2 \times 10^{8} \mathrm{~cm} \mathrm{~s}^{-1}$. The jet is initially underpressured with respect to the hot ambient medium (shocked wind). This generates a strong conical recollimation shock in the jet soon after injection, followed by pinching. At $z \approx 3 \times 10^{19} \mathrm{~cm}$, the jet head reaches the shocked ISM, and its advance is stopped. The speed of the head is reduced to $2 \times 10^{7} \mathrm{~cm} \mathrm{~s}^{-1}$, and the jet is only able to propagate up to $z \approx 3.2 \times 10^{19} \mathrm{~cm}$ by the end of the simulation. The expansion of the bow shock during this phase is mainly radial (perpendicular to $z$ ), with an expansion to a distance of $4.3 \times 10^{19} \mathrm{~cm}$ from the jet axis (outside the frame of the image). The reverse shock 

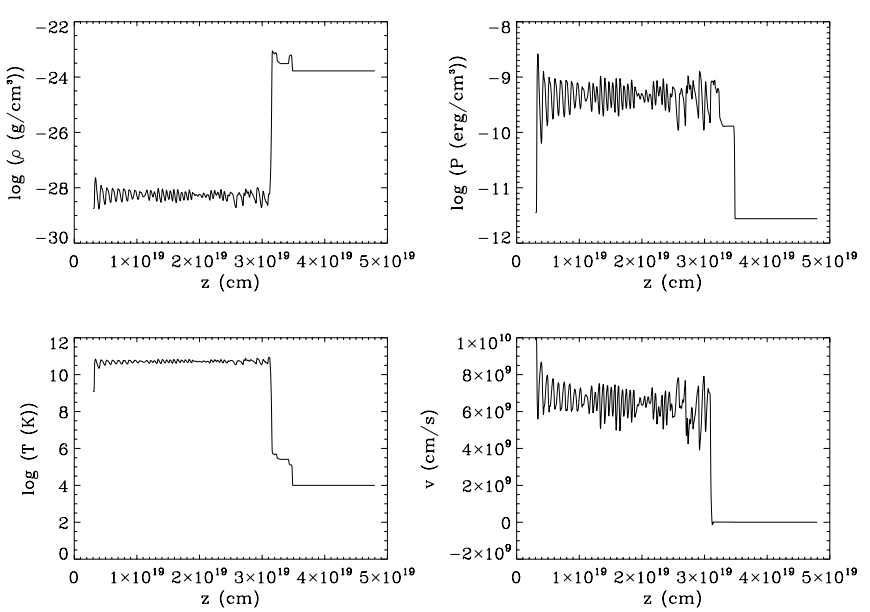

Fig. 2. Axial cuts of density (top, left), pressure (top, right), temperature (down, left), and velocity (down, right) at the last snapshot of the simulation in case $1\left(t_{\mathrm{src}}=8900 \mathrm{yr}\right)$. Units are cgs.

becomes stronger after the collision with the shocked ISM, and pinching is enhanced along the jet. By the end of the simulation, the SNR shock in the ISM has expanded farther, relaxing the density and pressure conditions that the jet finds at arrival, which makes it easier for the jet to carve its way across this region. At the given velocity, the jet would need about $3000 \mathrm{yr}$ more to complete its path through the shocked ISM.

In case 1 , the pressure in the cocoon and shocked medium (bow shock) regions is basically constant from the early stages of the simulation: $P_{\mathrm{s}} \approx 5 \times 10^{-10} \mathrm{erg} / \mathrm{cm}^{3}$. The shocked medium density starts increasing when the jet head reaches the ISM shocked by the $S N R$ from $3 \times 10^{-26} \mathrm{~g} / \mathrm{cm}^{3}$ to $5 \times 10^{-26} \mathrm{~g} / \mathrm{cm}^{3}$ during the period 4500-8900 yr. The temperature in the shocked ambient medium falls slowly during the whole run from $2 \times 10^{8}$ to $1.2 \times 10^{8} \mathrm{~K}$. The cocoon density increases from $3 \times 10^{-28} \mathrm{~g} / \mathrm{cm}^{3}$ to $7 \times 10^{-28} \mathrm{~g} / \mathrm{cm}^{3}$ during the first $2000 \mathrm{yr}$ owing to the jet low pressure with respect to the ambient medium, and remains fairly constant afterwards because of the recollimation and pinching shocks. For the same reason, the temperature in the cocoon decreases from $3 \times 10^{10}$ to $10^{10} \mathrm{~K}$ during these first $2000 \mathrm{yr}$, and becomes constant from that moment on. Turbulence and mixing of jet and external material takes place in the cocoon. Turbulence follows, down to the scales allowed by the simulation resolution, a spectrum similar to Kolmogorov, and the entrained external material actually dominates the mass content of the cocoon. The axial cuts of density, pressure, temperature, and velocity in the last frame, presented in Fig. 2, show the rich structure in the jet after it has been pinched by the ambient pressure, with plenty of shocks, up to the point where the jet material reaches the strong reverse shock in the interaction with the ISM shocked by the $S N R$. The recollimation and pinching shocks appear early in the simulation given the low initial lateral pressure of the jet with respect to the medium, with the pinching structures getting enhanced when the jet head reaches the shocked ISM. The maps for the density, pressure, temperature, and axial velocity at the last snapshot (8900 yr) of the evolution are shown in Fig. 3. The temporal evolution of the average cocoon and bow-shock density, pressure, temperature, and velocity are shown in Fig. 4. The temporal evolution of the jet head position and the mean radius of the bow shock are shown in Fig. 5.

The simulation of case 2 was run during 9800 yr of evolution. The jet crosses the shocked wind region from injection at
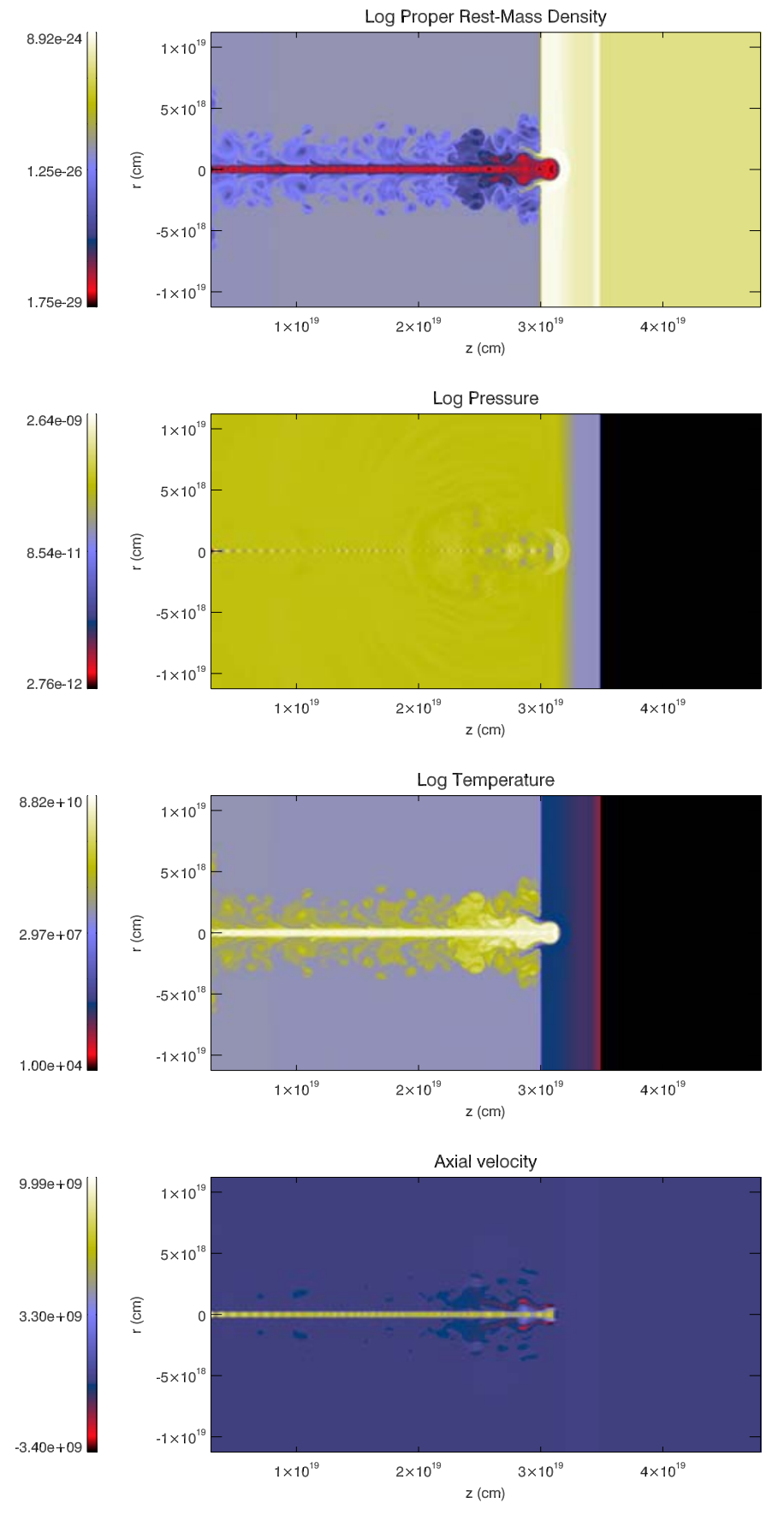

Fig. 3. From top to bottom, maps of density, pressure, temperature, and axial velocity at the last snapshot of the simulation in case $1\left(t_{\mathrm{src}}=\right.$ $8900 \mathrm{yr})$. Units are cgs.

$z \approx 3 \times 10^{18} \mathrm{~cm}$ to the contact discontinuity with the shocked ISM at $z \approx 2 \times 10^{19} \mathrm{~cm}$, at $1500 \mathrm{yr}$ after injection, at a mean velocity of $v_{\mathrm{h}} \approx 3.5 \times 10^{8} \mathrm{~cm} \mathrm{~s}^{-1}$. As in case 1 , the jet is initially underpressured and collimated by the ambient medium, which produces pinching oscillations along the jet body. After reaching the shocked ISM, the jet head gets flattened by the highdensity wall. At that point, the pressure in the bow-shock and cocoon regions starts to decrease more slowly with time. The same happens with the density in the cocoon, whereas the density of the shocked ambient starts to increase, since the head of the jet interacts with a denser medium. The backflow expands sideways, filling the simulated region, whereas the head of the 

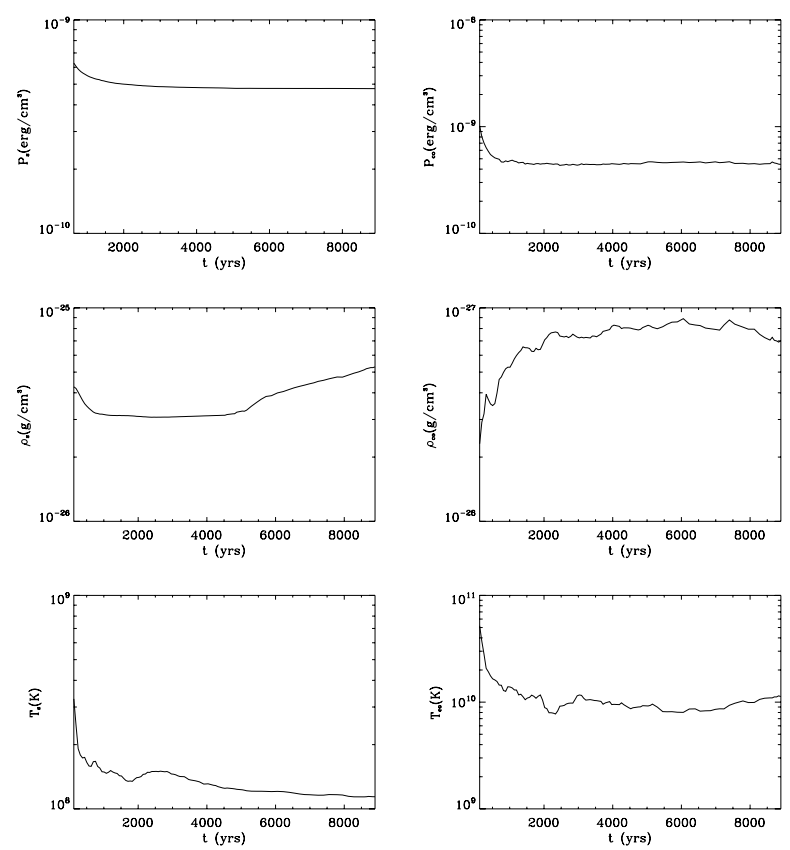

Fig. 4. Evolution of pressure (top), density (middle), and temperature (bottom) in the bow shock (left) and cocoon (right) regions for case 1. Units are cgs.
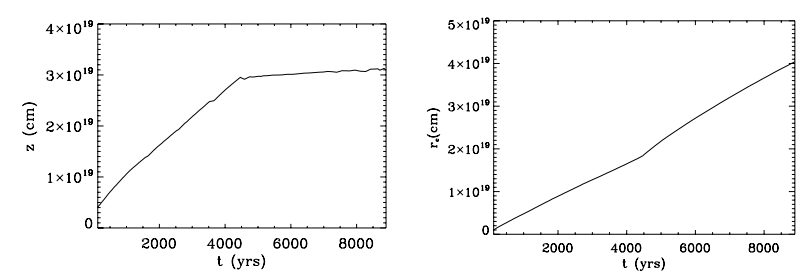

Fig. 5. Position of the jet head (left) and mean radius of the bow-shock region (right) for case 1. The bow shock has already left the shown grid by the end of the simulation. Units are cgs.

jet acts as a drill on the shocked ISM region owing to its much higher pressure and crosses it at $t \approx 4300 \mathrm{yr}$ after injection. Thus, it needs $3000 \mathrm{yr}$ to cross this small shell. As in case 1, the internal structure of the jet becomes very rich thanks to the interaction between the previous pinching patterns and the backward moving waves produced at the shock of the jet flow with the stagnation point, at $z \approx 2.4 \times 10^{19} \mathrm{~cm}$. The axial cuts of the jet, presented in Fig. 6, show this effect and the increase in the temperature and decrease in the velocity after each collimation shock, in which strong pressure jumps are produced. After the jet head crosses the shocked ISM layer, it propagates in the ISM with a supersonic speed of $\approx 2.4 \times 10^{7} \mathrm{~cm} \mathrm{~s}^{-1}$, an order of magnitude smaller than the one in the shocked wind region. The maps for the density, pressure, temperature, and axial velocity at the last snapshot (9800 yr) of the evolution are shown in Fig. 7. The temporal evolution of the average cocoon and bow-shock density, pressure, temperature, and velocity are shown in Fig. 8. The temporal evolution of the jet head position and the mean radius of the bow shock are shown in Fig. 9. Overall, the jet evolution of case 2 is similar to case 1 , with the cocoon also affected by turbulence and strong mixing.

Simulation 3 was run during $\approx 2000 \mathrm{yr}$. The jet was injected in the grid in the wind region at $z=1.25 \times 10^{18} \mathrm{~cm}$. When the jet reaches the shocked wind region, at $z=8 \times 10^{18} \mathrm{~cm}$, it gets
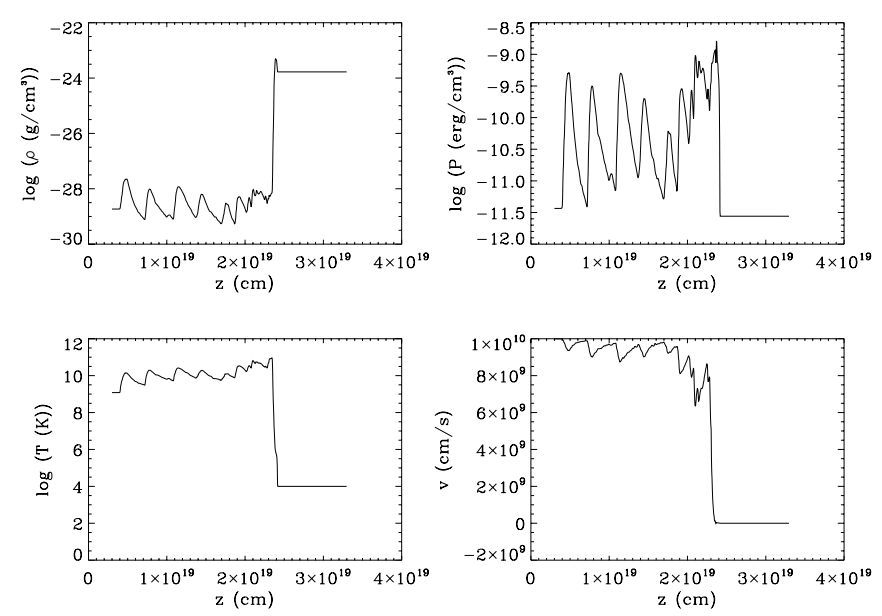

Fig. 6. Same as in Fig. 2 but for case $2\left(t_{\mathrm{src}}=9800 \mathrm{yr}\right)$.

the impact from the shocked wind that has been deflected by the propagation of the binary system in the ISM (see Fig. 1, bottom). This wind is enough in the slab simulation to distort the bow shock of the jet and disrupt the latter as follows. After crossing the wind/shocked wind discontinuity, the bow shock is deviated to the right from injection in the images by the shocked wind coming from top to bottom. The backflow is also deviated in this direction dy the generated pressure gradient. This generates an excess of backflow material in the unshocked wind region to the right of the jet, resulting in a deviation of the jet to the left. The combination of this process with the shocks generated at the different discontinuities destroys the jet even inside the wind region. When the head of the jet reaches the shocked ISM region after $300 \mathrm{yr}$, at $z=10^{19} \mathrm{~cm}$, the jet does not have enough thrust to penetrate this layer, and all the injected flow is thus converted into a backflow that fills both the unshocked and the shocked wind regions. The combination of disruption and advection does not allow the jet to reach the normal ISM regardless of the time elapsed, and thus jets with the given power will be trapped inside the wind/shocked wind region and advected backwards with respect to the system motion. The maps for the density, pressure, temperature, and axial velocity, in the last snapshot (2000 yr) and after $300 \mathrm{yr}$ of evolution, are shown in Figs. 10-13.

\section{Nonthermal activity}

To estimate what may be the potential nonthermal luminosity of the radiation produced when the jet head propagates within the SNR or the shocked stellar wind, we used a model for the cocoon emission similar to the one presented in Bordas et al. (2009). In short, the model assumes the cocoon as an homogeneous emitter, which is true to first order provided that the flow is roughly sonic. Our simulations show turbulent motion and jet/medium mixing in the cocoon, and the flow becomes trans-sonic far from the jet tip, but we neglect these effects at this stage. In the radiation calculations, following Bordas et al. (2009), the magnetic field $(B)$ energy density $\left(u_{\mathrm{B}}\right)$ was assumed to be a $10 \%$ of the matter energy density, and the nonthermal injection luminosity, $1 \%$ of the jet power. For an optically thin emitter, the radiation luminosity will just be proportional to this fraction. Postshock magnetic field values close to, or not far below, equipartition are expected if the jet keeps a non-negligible fraction of its total energy as magnetic energy, which is a reasonable assumption provided that jets are magnetically launched (e.g. Komissarov et al. 2007; 

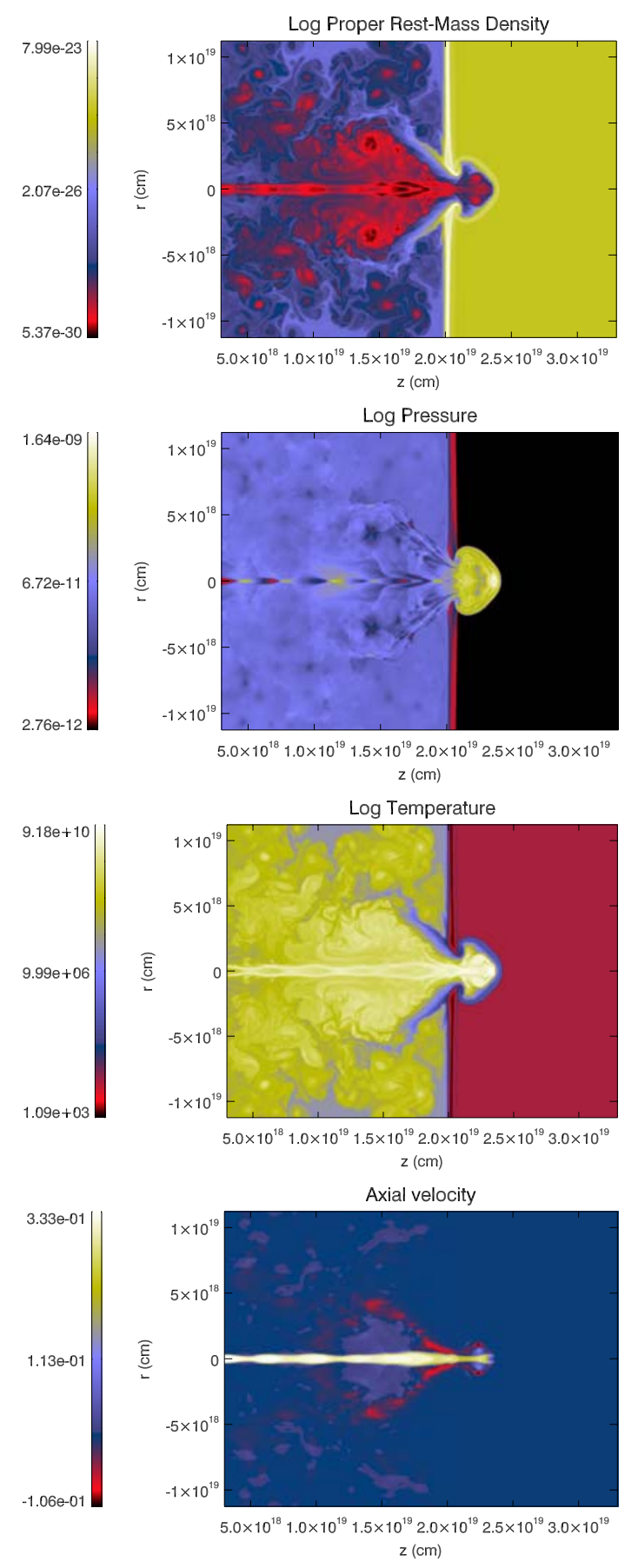

Fig. 7. Same as in Fig. 3 but for case $2\left(t_{\mathrm{src}}=9800 \mathrm{yr}\right)$.

Spruit 2010). Unlike in Bordas et al. (2009), here only the cocoon has been considered as a powerful emitter, since the recollimation and reverse shocks suffered by the jet are strong, but the bow shock is too weak to accelerate particles. The results for the cocoon radiation are presented in Sect. 4.1, in which we focus only on electrons, given the low cocoon densities that prevent efficient gamma-ray production from proton-proton interactions. These low densities also imply that relativistic Bremsstrahlung will be inefficient there.

When the jet head impacts the shocked ISM thin shell, the highest energy particles accelerated in the cocoon region may reach this shell, which in case 2 reaches densities $n_{\mathrm{ISMs}} \sim$ $100 \mathrm{~cm}^{-3}$. Given the relativistic Bremsstrahlung (electrons) and proton-proton interaction (if protons are also accelerated)
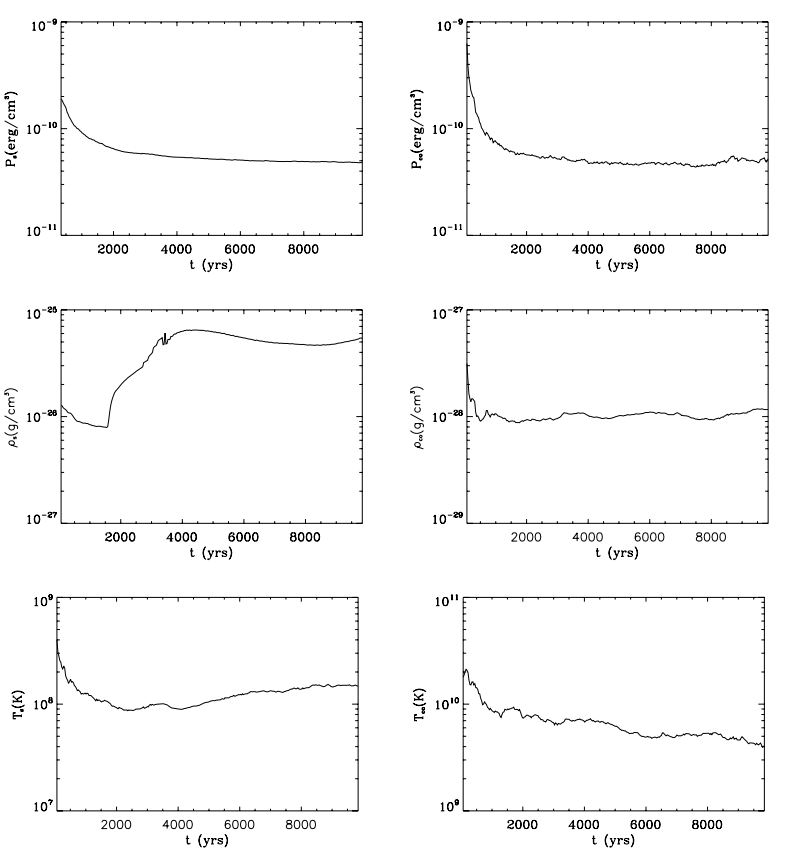

Fig. 8. Same as in Fig. 4 but for case 2.
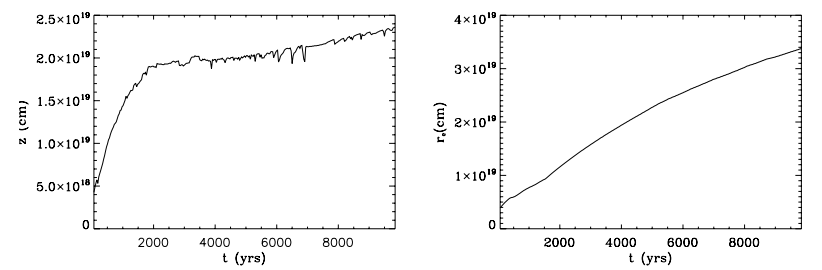

Fig. 9. Same as in Fig. 5 but for case 2.

timescales and the source age, about $1-10 \%$ of the luminosity in relativistic particles reaching that shell could be radiated at very high energies. We note that denser media would increase the efficiency, which is simply $\propto n_{\mathrm{ISMs}}$ (if not in the saturation regime).

For the case when the jet has reached the undisturbed ISM, if it is not disrupted, the nonthermal behavior can be described as in Bordas et al. (2009). This would apply for instance to the emission produced during the second half of case 2 simulation. We have not consider particle acceleration in the turbulent and sheared flow in the recollimated jet and cocoon regions, but stochastic and shear acceleration may be relevant there (see Rieger et al. 2007, and references therein). Some thermal radiation may be produced in the shocked ISM. This thermal component would tend to be quite extended and, given the shock velocities, peak in the UV.

We do not consider case 3 here since jet full disruption makes conditions seem less suitable for particle acceleration. It might be that stochastic and shear acceleration may still take place in the jet-disrupted medium, although estimating the nonthermal emission for this case requires an in-depth study that is beyond the scope of this work.

\subsection{Cocoon emission: cases 1 and 2}

The simulation results are used to characterize the physical properties of the shocked regions. The position and velocity of the bow shock, the internal energy density $\left(u_{\mathrm{th}}\right.$; and hence $\left.u_{\mathrm{B}}=0.1 u_{\mathrm{th}}\right)$, the temperature, and the rest mass density are 
V. Bosch-Ramon et al.: The termination region of HMMQ jets
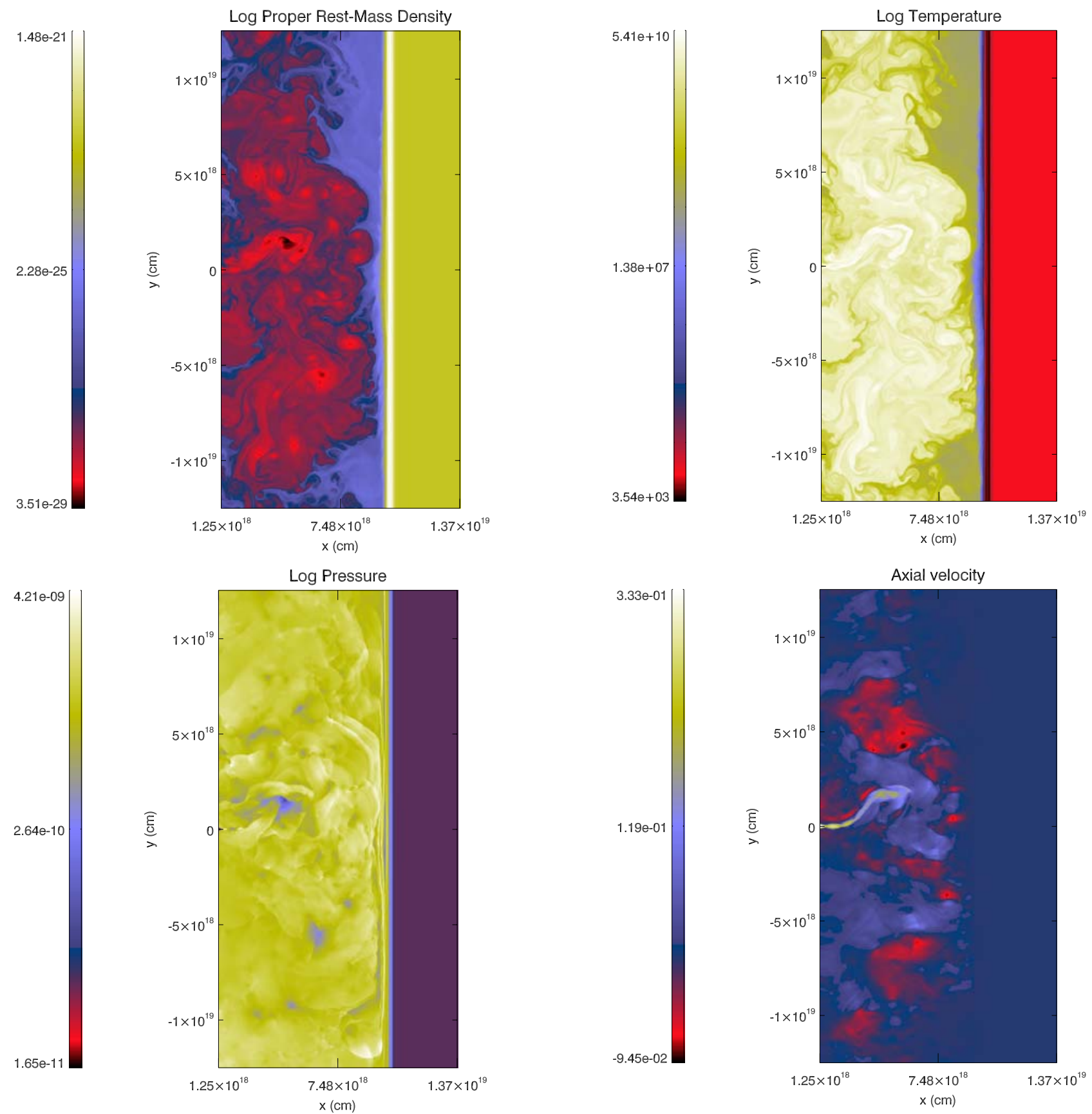

Fig. 10. Maps of density (top) and pressure (bottom) at the last snapshot of the simulation for case $3\left(t_{\mathrm{src}} \approx 2000 \mathrm{yr}\right)$. The shocked wind moves from top to bottom.

used at any source age to account for the evolution of the particle energy distribution, which is affected by both adiabatic and radiative losses: relativistic Bremsstrahlung (negligible), inverse Compton (minor) and synchrotron (dominant). For the IC emission, the radiation field provided by the companion star dominates the $\mathrm{CMB}$. The star is taken to be a bright $\mathrm{O}$, with luminosity $10^{39} \mathrm{erg} \mathrm{s}^{-1}$ and temperature $4 \times 10^{4} \mathrm{~K}$. At a distance of $\sim 10 \mathrm{pc}$, the energy density ratio is $u_{*} / u_{\mathrm{CMB}} \sim 7$, increasing towards the central engine like $\propto z^{-2}$.

We assumed that the recollimation and reverse shocks accelerate particles, simplifying them as just one accelerator with average properties. The shock velocity was taken to be $v_{\mathrm{s}} \sim v_{\mathrm{j}}$, which yields an acceleration efficiency $\dot{E} \sim$ $(1 / 2 \pi)\left(v_{\mathrm{s}} / c\right)^{2} q B c \approx 0.02 \times q B c$ (e.g. Drury 1983). As

Fig. 11. Maps of temperature (top) and axial velocity (bottom) at the last snapshot of the simulation for case $3\left(t_{\mathrm{src}} \approx 2000 \mathrm{yr}\right)$. The shocked wind moves from top to bottom.

mentioned before, the assumed nonthermal injection luminosity is $1 \%$ that of the jet, i.e. $3 \times 10^{34} \mathrm{erg} \mathrm{s}^{-1}$, and the magnetic-tointernal energy density ratio is 0.1 . This gives a $B$-strength of about $\sim 4-6 \times 10^{-5} \mathrm{G}$ depending on $t_{\text {src }}$. These $B$-values are one order of magnitude higher than those inferred by Tudose et al. (2006) and Safi-Harb \& Petre (1999) for the jet termination regions in Circinus X-1 (a low-mass microquasar) and SS 433, respectively. On the other hand, the ages of these sources are also about one order of magnitude older than those considered here, and thus a lower magnetic field is naturally expected in the former cases. With the adopted magnetic field, synchrotron losses are dominant in the cases studied here. For the conditions assumed, maximum energies are typically $\sim 10^{3} \mathrm{TeV}$. We notice that the adopted nonthermal fraction is quite conservative, and 

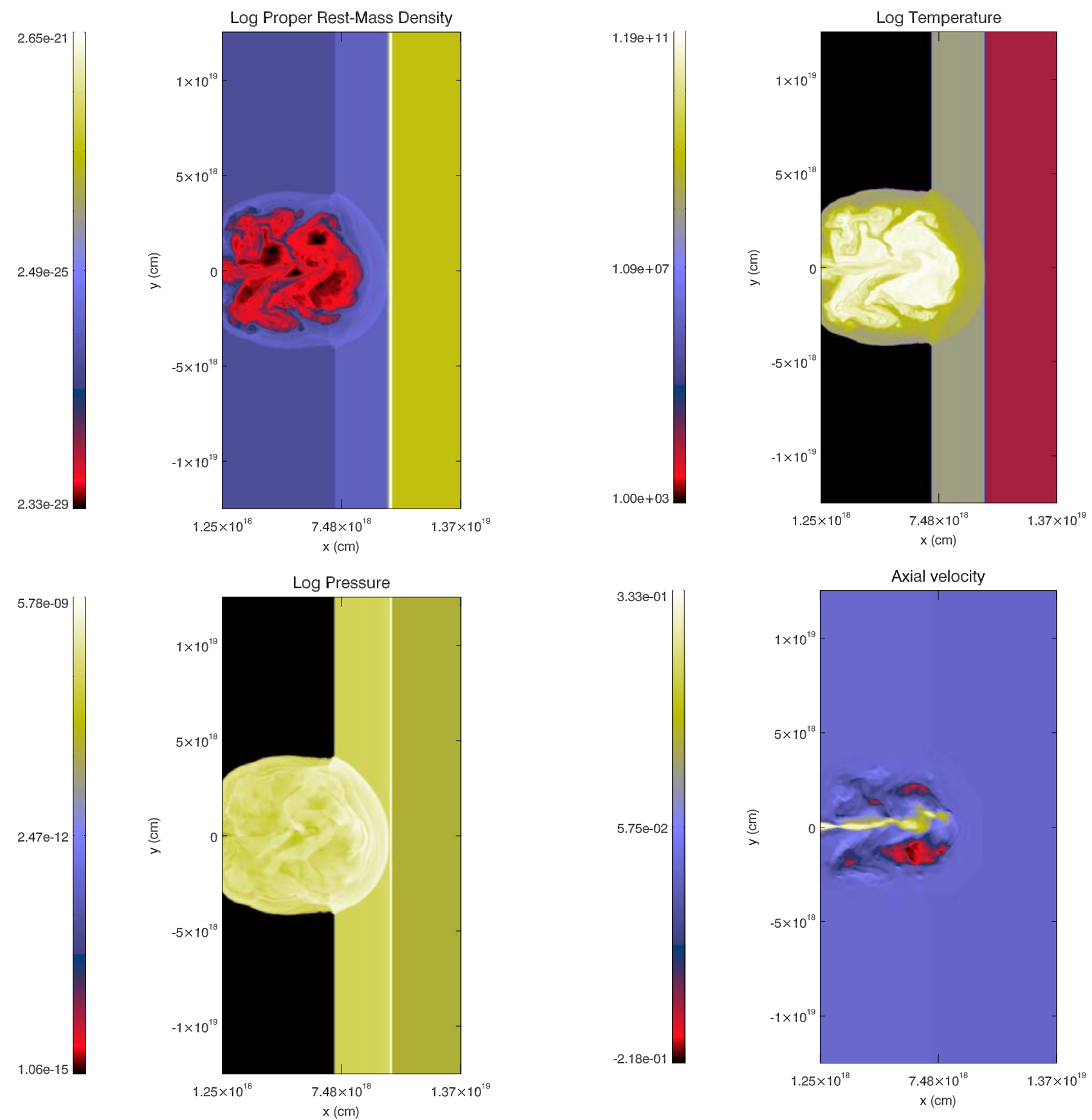

Fig. 12. Same as in Fig. 10 but for a source age of $300 \mathrm{yr}$.

there is room to increase it. As a reference, the values of the radiation model parameters are listed in Table 2 for cases 1 and 2 when the source age is $\sim 10^{4} \mathrm{yr}$.

The spectral energy distributions (SED) of the nonthermal synchrotron and IC emission for cases 1 and 2, for evolution times $t_{\mathrm{src}}=10^{3}, 3 \times 10^{3}$, and $9 \times 10^{3} \mathrm{yr}$, are shown in Fig. 14 . Depending on the age of the source and the case, and given that $u_{\mathrm{B}} \gg u_{*}$, the synchrotron luminosity can be between $\sim 10$ 1000 times higher than the IC one. This difference is more pronounced in case 1 . This is a result of the smaller amount of energy pumped by the stellar wind compared to that of an $S N R$, which leads to lower pressure and therefore lower $B$ in case 2 . The IC dominance may be only possible if the $B$-strength were well below equipartition. At the end of the simulations $\left(t_{\mathrm{src}}=9 \times 10^{3} \mathrm{yr}\right)$, the synchrotron fluxes are, for a $3 \mathrm{kpc}$ distance

Fig. 13. Same as in Fig. 11 but for a source age of $300 \mathrm{yr}$.

source, of $\sim 20 \mathrm{mJy}$ (case 2) and $100 \mathrm{mJy}$ (case 1 ), and $\sim 4 \times 10^{-12}$ (case 2) and $7 \times 10^{-12} \mathrm{erg} \mathrm{cm}^{-2} \mathrm{~s}^{-1}$ (case 1), in radio and X-rays, respectively. The IC emission yields fluxes of $\sim 6 \times 10^{-14}$ (case 1) and $10^{-13} \mathrm{erg} \mathrm{cm}^{-2} \mathrm{~s}^{-1}$ (case 2), and $\sim 5 \times 10^{-13}$ (case 1) and $3 \times 10^{-13} \mathrm{erg} \mathrm{cm}^{-2} \mathrm{~s}^{-1}$ (case 2), in the ranges $0.1-100 \mathrm{GeV}$ and $>100 \mathrm{GeV}$, respectively. These fluxes might be in principle significantly higher, since the nonthermal fraction is not much constrained.

The cocoon is in pressure equilibrium with the medium. Since the dynamical timescales of the SNR/ISM and wind/ISM interaction structures are longer than the structures generated by the jet, the magnetic field is likely to be approximately constant with time as long as the jet remains inside the SNR/ or the wind/ISM structure. In this context, the radio emission grows with the source age due to particle accumulation, but the $\mathrm{X}$-ray fluxes do not change much, because X-ray synchrotron 

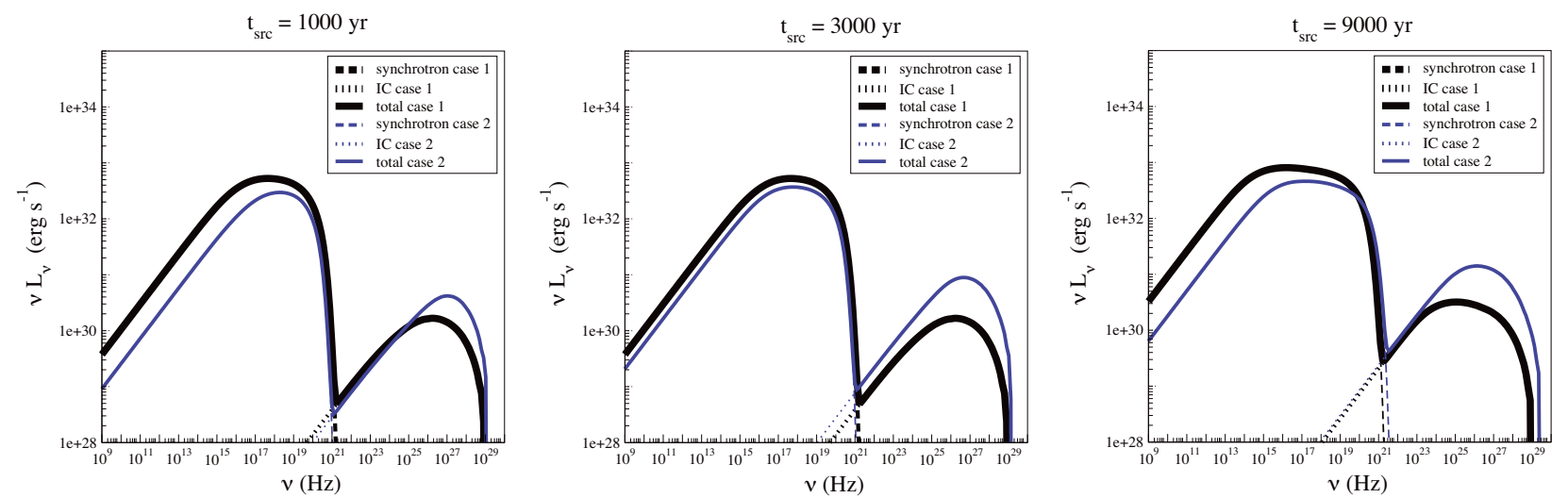

Fig. 14. Synchrotron and IC SEDs for cases 1 (black/thick lines) and 2 (blue/thin lines) computed for $t_{\mathrm{src}}=10^{3}($ left $), t_{\mathrm{src}}=3 \times 10^{3}(\mathrm{middle})$, and $t_{\mathrm{src}}=9 \times 10^{3} \mathrm{yr}($ right $)$.

Table 2. Model parameters for the shell and cocoon for three different source ages.

\begin{tabular}{lc}
\hline \hline Parameter & Value \\
\hline Jet power [erg s & -1] \\
Nonthermal power fraction & $3 \times 10^{36}$ \\
Stellar field energy density (case 1) $\left[\mathrm{erg} \mathrm{cm}^{-3}\right]$ & 0.01 \\
Stellar field energy density (case 2) $\left[\mathrm{erg} \mathrm{cm}^{-3}\right]$ & $3.1 \times 10^{-12}$ \\
Magnetic field (case 1) [G] & $5.2 \times 10^{-12}$ \\
Magnetic field (case 2) [G] & $4.1 \times 10^{-5}$ \\
Acceleration efficiency & $1.4 \times 10^{-5}$ \\
Injection power-law index & 0.02 \\
Maximum energy (case 1) & 2.1 \\
Maximum energy (case 2) & $10^{3} \mathrm{TeV}$ \\
\hline
\end{tabular}

emitting electrons have time to cool down from quite early times. Concerning IC, the increase in the available cooling time owing to longer $t_{\mathrm{src}}$ compensates for the dilution of the stellar photon field (and subsequent reduction of the emission efficiency) produced by the increase in cocoon size. This renders fairly constant total IC fluxes, although the spectrum below $\sim 1 \mathrm{TeV}$ becomes softer with time. In Fig. 15, we present the evolution of the specific flux at $5 \mathrm{GHz}$, and the bolometric fluxes between $1-10 \mathrm{keV}$, $0.1-100 \mathrm{GeV}$, and $>100 \mathrm{GeV}$ up to $10^{4} \mathrm{yr}$. In general, all the lightcurves show a smooth increase for the considered times.

From the morphological point of view, the sources discussed here would appear extended on scales of $\sim 10^{\prime}(d / 3 \mathrm{kpc})^{-1}$ in radio, soft X-rays, and high-energy gamma-rays. At hard $\mathrm{X}$-rays and $\sim 1 \mathrm{TeV}$, the emission would be rather concentrated close to the jet reverse shock and possibly the jet recollimation shocks, since the emitting electrons, with lifetimes $\approx 1.3 \times 10^{2}\left(B / 10^{-4} \mathrm{G}\right)^{-2}(E / 10 \mathrm{TeV})^{-1} \mathrm{yr}$, may not have time to fill the cocoon.

\section{Discussion}

In the light of our results, we briefly discuss here the two HMMQs with very clear evidence of jet interaction with the ISM: SS 433 (case 1) and Cygnus X-1 (case 2). In the case of SS 433, the jet is strongly interacting with its supernova remnant (e.g. Zealey et al. 1980). This source is therefore a clear example of a jet/SNR interaction, but the luminosity of the jet, $\sim 10^{40} \mathrm{erg} \mathrm{s}^{-1}$, is much higher than the value adopted here. Nevertheless, there is evidence of nonthermal radio and X-ray
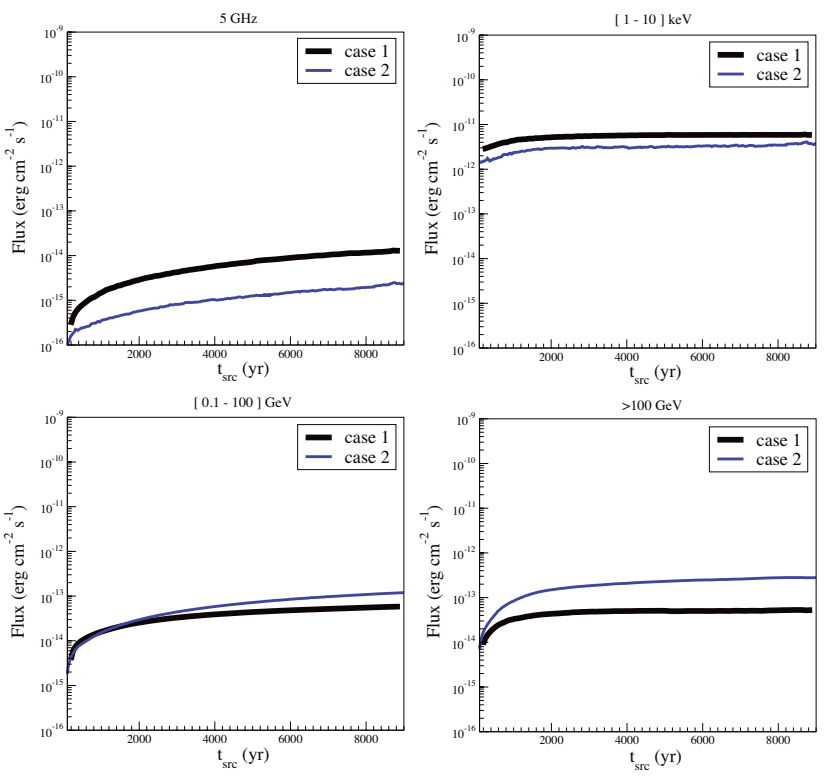

Fig. 15. Evolution of the computed fluxes at $5 \mathrm{GHz}$ (top, left), $1-10 \mathrm{keV}$ (top, right), 0.1-100 GeV (bottom, left), and $>100 \mathrm{GeV}$ (bottom right) up to $10^{4} \mathrm{yr}$, for cases 1 (black/thick lines) and 2 (blue/thin lines).

emission in the $S N R /$ jet interaction regions of SS 433, which implies that particle acceleration is taking place there (e.g. Safi-Harb \& Petre 1999), and possibly also gamma-rays, although at present only upper-limits are available (see Bordas et al. 2010, and references therein). It is worth noting that the interaction of an SS 433-like jet with its external medium has been detected even in nearby galaxies (e.g. Pakull et al. 2010).

Concerning Cygnus X-1, there is a thin emission region that seems to trace the bow shock formed by the jet in an inhomogeneous ISM (the counter-jet is not detected; see, e.g., Gallo et al. 2005). An $S N R /$ jet interaction can indeed be discarded, because the $S N R$ apparently was very weak, as reported by Mirabel \& Rodrigues (2003). The structure is elongated, so a shocked wind/jet origin seems unlikely from observations, although the wind energetics $\left(\sim 10^{36}-10^{37} \mathrm{erg}^{-1}\right)$ plus medium inhomogeneity might be a possibility, albeit remote. On the other hand, the deprojected distance of the bow shock to the system is about 10 pc (Gallo et al. 2005), far enough for the jet head to have already left the shocked wind/ISM region. As noted above, the non-detection of emission from the cocoon region may be related to jet disruption. In such a case, radiation may 
still be generated from particles accelerated via stochastic or shear acceleration or the jet recollimation $\operatorname{shock}(\mathrm{s})$, which is expected even if the jet disrupts at farther distances (see Bordas et al. 2011, for a similar situation in extragalactic sources). The bow shock in Cygnus X-1 does not show clear signatures of the system proper motion, which can be explained by the low peculiar velocity of the source with respect to its birth association, Cygnus OB3 (Mirabel \& Rodrigues 2003), plus a relatively young jet age (Gallo et al. 2005).

Other high-mass systems may be good candidates for studying the interaction of the jet with its surroundings. Cygnus X-3, for instance, has a powerful jet, and radiation has been detected in the surroundings of this source (Heindl et al. 2003; Sánchez-Sutil et al. 2008), although the nonthermal nature of this emission and its relation to Cygnus X-3 still need to be confirmed. Hints of extended X-ray emission have been also found in LS I +61 303 (Paredes et al. 2007; see however Rea et al. 2010), but the microquasar nature of this source is disputed (see, e.g., Bosch-Ramon \& Khangulyan 2009, and references therein).

The number of powerful HMMQs interacting with the environment may be on the order of 10 in our Galaxy ${ }^{1}$ (see, e.g., Perucho et al. 2010). The small number of sources and their preferred location in the inner regions of the Galaxy (Bosch-Ramon et al. 2005) make their detection difficult because of strong local/foreground absorption of radio and soft $\mathrm{X}$-ray emission. Diffuse hard X-rays, clustered in the acceleration regions (recollimation and reverse shocks, and possibly the bow shock generated in the jet-ISM interaction), may be better probes for unveiling young HMMQs. Very high-energy radiation could be detected from jet/medium interactions by forthcoming Cherenkov instrumentation (e.g. CTA), which does not suffer from strong gamma-ray background contamination. It could be, however, that most HMMQs would be old and fast enough to have a well-developed bow shock driven by the proper motion of the system. In such a case, the jet would be destroyed and mixed with material from the system motion-driven bow shock, and its large-scale radiation, if any, could be hard to detect.

\section{Summary}

In the present work, we have explored different possibilities for propagating of a HMMQ jet, assuming different initial times for the jet activity to start with respect to the formation of the HMXB: (i) shortly ( 40000 yr) after the $S N R$ explosion of the progenitor of the compact object; (ii) at epochs $\left(\sim 10^{5} \mathrm{yr}\right)$ at which only a spherical wind/ISM shock is present; and (iii) at the stages when the system peculiar motion bow shapes the wind/ISM shock to create strong shocked wind convection backwards $\left(>10^{5} \mathrm{yr}\right)$. For the two first cases, the jet needs about $10^{4} \mathrm{yr}$ to reach the unperturbed ISM, a period much shorter than the potential duration of an HMMQ, which may be up to the lifetime of the remaining massive star, of $\sim 10^{6} \mathrm{yr}$. For the third case, the jet gets disrupted and mixed with the shocked stellar wind, and advected away from the stellar bow shock, in just $\sim 10^{3} \mathrm{yr}$. For the cases in which the jet reaches the normal ISM, its propagation can be described as in Bordas et al. (2009), although the impact of crossing the shocked wind/SNR ejecta/shocked ISM can lead to jet disruption, in a similar way to FRI jets (e.g. Perucho \& Martí 2007). When the jet is embedded in the hot shocked wind/SNR ejecta, significant nonthermal emission can be generated in the cocoon region, mainly in radio and $\mathrm{X}$-rays, but also gamma rays. Hard X-rays and very high-energy photons may be the best probes for unveiling the presence of microquasar jets interacting with their environment.

Acknowledgements. The authors thank the referee, Philip Hughes, for his constructive and very encouraging comments. The research leading to these results has received funding from the European Union Seventh Framework Program (FP7/2007-2013) under grant agreement PIEF-GA-2009-252463 V.B.-R. and P.B. acknowledge support by the Ministerio de Educación y Ciencia (Spain) under grant AYA 2007-68034-C03-01, FEDER funds. M.P. acknowledges support from a "Juan de la Cierva" contract of the Spanish "Ministerio de Ciencia y Tecnología" and from the Spanish "Ministerio de Educación y Ciencia" through grants AYA2007-67627-C03-01, CSD2007-00050, and AYA2007-67752-C0302. P.B. acknowledges support from the German Federal Ministry of Economics and Technology through DLR grant 50 OG 1001. P.B. also acknowledges the excellent working conditions at the INTEGRAL Science Data Center. The authors acknowledge the "Servei d'Informàtica" of the "Universitat de València" for the computing time allocated for this project in "Tirant".

\section{References}

Blandford, R. D., \& McKee, C. F. 1976, Physics of Fluids, 19, 1130 Bordas, P., Bosch-Ramon, V., Paredes, J. M., \& Perucho, M. 2009, A\&A, 497, 325

Bordas, P., Bosch-Ramon, V., \& Paredes, J. M. 2010, IJMPD, 19, 749

Bosch-Ramon, V., Romero, G. E., \& Paredes, J. M. 2005, A\&A, 429, 267

Bordas, P., Bosch-Ramon, V., \& Perucho, M. 2011, MNRAS, in press [arXiv: astro-ph/1011.1653]

Bosch-Ramon, V., \& Khangulyan, D. 2009, Int. J. Mod. Phys. D, 18, 347

Castor, J., McCray, R., \& Weaver, R. 1975, ApJ, 200, L107

Drury, L. O. 1983, Rep. Prog. Phys., 46, 973

Fanaroff, B. L., \& Riley, J. M. 1974, MNRAS, 167, 31

Gallo, E., Fender, R., Kaiser, C., et al. 2005, Nature, 436, 819

Heindl, W. A., Tomsick, J. A., Wijnands, R., \& Smith, D. M. 2003, ApJ, 588, L97

Heinz, S., Grimm, H. J., Sunyaev, R. A., \& Fender, R. P. 2008, ApJ, 686, 1145 Kaiser, C. R., \& Alexander, P. 1997, MNRAS, 286, 215

Komissarov, S. S., Barkov, M. V., Vlahakis, N., \& Königl, A. 2007, MNRAS, 380,51

Martí, J., Rodríguez, L. F., Mirabel, I. F., \& Paredes, J. M. 1996, A\&A, 306, 449

Mirabel, I. F., \& Rodríguez, L. F. 1999, ARA\&A, 37, 409

Mirabel, I. F., \& Rodrigues, I. 2003, Science, 300, 1119

Pakull, M. W., Soria, R., \& Motch, C. 2010, Nature, 466, 209

Paredes, J. M., Ribó, M., \& Bosch-Ramon, V. 2007, ApJ, 2007, ApJ, 664, L39

Perucho, M., \& Martí J. M. 2007, MNRAS, 382, 526

Perucho, M., \& Bosch-Ramon, V. 2008, A\&A, 482, 917

Perucho, M., Bosch-Ramon, V., \& Khangulyan, D. 2010, A\&A, 512, L4

Puls, J., Sundqvist, J. O., Najarro, F., \& Hanson, M. M. 2009, AIPC, 1171, 123

Rea, N., Torres, D. F., \& van der Klis, M. 2010, MNRAS, 405, 2206

Ribó, M. 2005, in Future Directions in High Resolution Astronomy: The 10th Anniversary of the VLBA (ASPC, 2005) 340, 421

Rieger, F. M., Bosch-Ramon, V., \& Duffy, P. 2007, Ap\&SS, 309, 119

Russell, D. M., Fender, R. P., Gallo, E., \& Kaiser, C. R. 2007, MNRAS, 376 1341

Safi-Harb, S., \& Petre, R. 1999, ApJ, 512, 784

Sánchez-Sutil, J. R., Martí, J., Combi, J. A., et al. 2008, A\&A, 479, 523

Spruit, H. C. 2010, LNP, 794, 233

Tudose, V., Fender, R. P., Kaiser, C. R., et al. 2006, MNRAS, 372, 417

Velázquez, P. F., \& Raga, A. C. 2000, A\&A, 362, 780

Zavala, J., Velázquez, P. F., Cerqueira, A. H., \& Dubner, G. M. 2008, MNRAS, 387,839

Zealey, W. J., Dopita, M. A., \& Malin, D. F. 1980, MNRAS, 192, 731

\footnotetext{
1 We do not consider here the impact of microquasars as a class, including low-mass sources, on the ISM properties (see, e.g., Heinz et al. 2008), but focus on the specific case of jet/environment interaction in HMMQs.
} 